\title{
The Production of "Braids" in Saturn's F Ring
}

\section{Jack J. Lissauer and Stanton J. Peale (UCSB),}

Two models are presented to explain the "braided" structure observed in some Voyager images of Saturn's $F$ ring. $\Lambda$ braided pattern can be produced from an initially uniform, circular, narrow ring, which orbits past a nearby satellite (resulting in a wavy pattern) and then is doubled back upon itself due to gravitational accelerations from an embedded moonlet (Figure 1). Viewed from the frame which rotates with the moonlet's orbital angular velocity, the trajectories of the ring particles traverse one end of the now classic horseshoe orbit. At least one (and probably both) of the F ring shepherding satellites is massive enough to produce the required wavy pattern; an icy moonlet 5-10 $\mathrm{km}$ in radius would be massive enough to subsequently cause the ring particles to horseshoe into irregular, short braided strands. Alternatively, if the F ring is composed of two well-separated strands prior to the passage of the shepherding moon, then the differing wavelengths of the wavy patterns induced in the two strands and the subscquent drift in relative phase can lead to a regular, lengthy braided pattern, without the need for an embedded moonlet. (Figure 2).

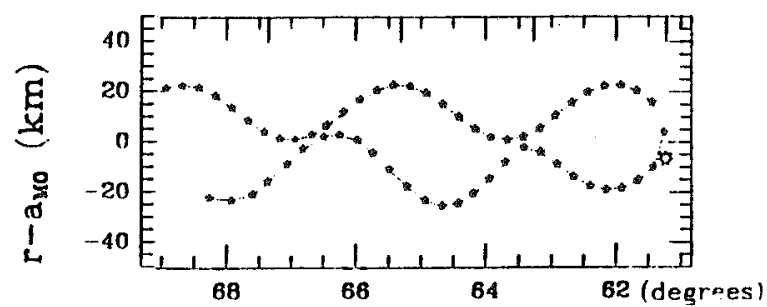

Fig. 1: Single strand Shepherd plus moonlet perturbations.

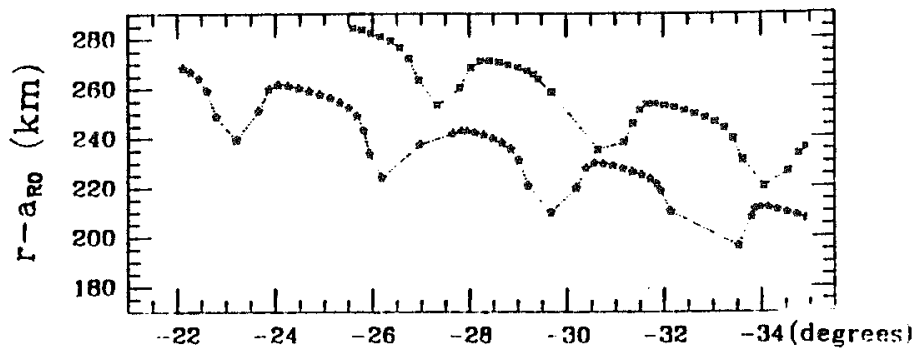

Fig. 2: Two strands, shepherding perturbations only. 